\title{
Fundamental Study of Optical Threshold Layer Approach Towards Double Exposure Lithography
}

\author{
Xinyu Gu ${ }^{a}$, Adam J. Berro ${ }^{b}$, Younjin Cho ${ }^{a}$, Kane Jen ${ }^{a}$, Saul Lee ${ }^{a}$, Tomoki Nagai ${ }^{a}$, Toshiyuki Ogata ${ }^{b}$, \\ William J. Durand ${ }^{\mathrm{a}}$, Arunkumar Sundaresan ${ }^{\mathrm{c}}$, Jeffrey R. Lancaster ${ }^{\mathrm{c}}$, Steffen Jockusch ${ }^{\mathrm{c}}$, Paul \\ Zimmerman $^{\mathrm{d}}$, Nicholas J. Turro ${ }^{\mathrm{c}}$ and C. Grant Willson ${ }^{\mathrm{a}, \mathrm{b}}$ \\ ${ }^{\mathrm{a}}$ Department of Chemical Engineering and ${ }^{\mathrm{b}}$ Department of Chemistry, The University of Texas at \\ Austin, Austin, TX 78712, USA; \\ ${ }^{c}$ Department of Chemistry, Columbia University, New York, NY 10027, USA; \\ ${ }^{\mathrm{d}}$ Intel Assignee to SEMATECH
}

\begin{abstract}
193 immersion lithography has reached its maximal achievable resolution. There are mainly two lithographic strategies that will enable continued increase in resolution. Those are being pursued in parallel. The first is extreme ultraviolet (EUV) lithography and the second is double patterning (exposure) lithography. EUV lithography is counted on to be available in 2013 time frame for $22 \mathrm{~nm}$ node ${ }^{[1]}$. Unfortunately, this technology has suffered several delays due to fundamental problems with source power, mask infrastructure, metrology and overall reliability ${ }^{[2]}$. The implementation of EUV lithography in the next five years is unlikely due to economic factors. Double patterning lithography (DPL) is a technology that has been implemented by the industry and has already shown the proof of concept for the $22 \mathrm{~nm}$ node ${ }^{[3]}$. This technique while expensive is the only current path forward for scaling with no fundamental showstoppers for the $32 \mathrm{~nm}$ and $22 \mathrm{~nm}$ nodes. Double exposure lithography (DEL) is being proposed as a cost mitigating approach to advanced lithography. Compared to DPL, DEL offers advantages in overlay and process time, thus reducing the cost-of-ownership $(\mathrm{CoO})^{[4][5]}$. However, DEL requires new materials that have a non-linear photoresponse. So far, several approaches were proposed for double exposure lithography, from which Optical Threshold Layer (OTL) was found to give the best lithography performance according to the results of the simulation ${ }^{[4][5]}$. This paper details the principle of the OTL approach. A photochromic polymer was designed and synthesized. The feasibility of the material for application of DEL was explored by a series of evaluations.
\end{abstract}

Keywords: Double Exposure Lithography, Optical Threshold Layer, azobenzene, side chain crystal polymer, poly(n-alkyl methacrylate), acid detector, barrier layer, diffusion switch, non-linear response

Advances in Resist Materials and Processing Technology XXVI, edited by Clifford L. Henderson, Proc. of SPIE Vol. 7273, 72731C · C 2009 SPIE · CCC code: 0277-786X/09/\$18 · doi: 10.1117/12.814298 


\section{INTRODUCTION}

\subsection{Principles of Optical Threshold Layer}

The main idea of the Optical Threshold Layer (OTL) approach involves a material that can receive a subthreshold exposure dose which is reversible. The typical analog of this type of behavior is the thermal phase change. However, the threshold response must be obtained with photons rather than heat because laser induced absorption produces insufficient heat to reach the threshold thermal dose for the phase change. Moreover, the introduction of the local temperature variation could disturb the carefully-controlled temperature within the exposure chamber and therefore affect the strict overlay alignment. However, phase change is still a type of threshold response that is promising. The question is whether the phase change can be accomplished by photo-induction instead of requiring the exposure tool output to perform heating on the materials. A reversible photo-induced structural change is potentially useful for realizing the threshold phase behavior since the phase transition temperature of the material is related to its structure. The phase transition temperature can be designed to shift from above ambient temperature down to below ambient temperature and shift back again upon irradiation at a different wavelengths, leading toward a reversible photoinduced phase change.

As a starting point, a trilayer OTL system was considered, where the OTL is sandwiched between two reactive substances, acting as a barrier layer. This barrier layer is initially impermeable to both substances. However, its permeability is switchable by UV irradiation. The regions that are exposed to the threshold dose undergo a structural change which induces an isothermal phase change. This allows the substance on one side to diffuse in an anisotropic manner through the barrier layer and react with the target species. The products of the reaction facilitate the pattern transfer. The barrier layer is then reverted back to its initial impermeable frozen phase before the subsequent exposure. The detailed mechanism schematic is shown in Fig. 1.

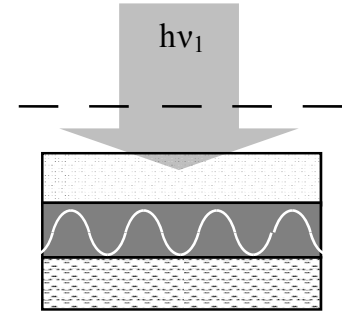

(1) 1st Exposure

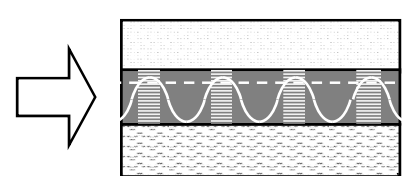

(2) Threshold Image

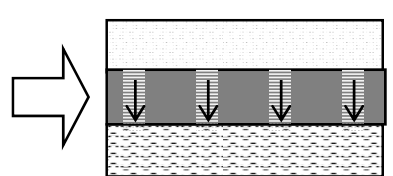

(3) Diffusion

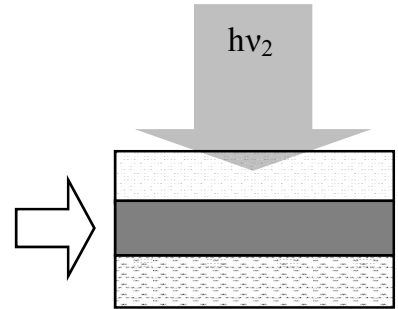

(4) Flood Exposure

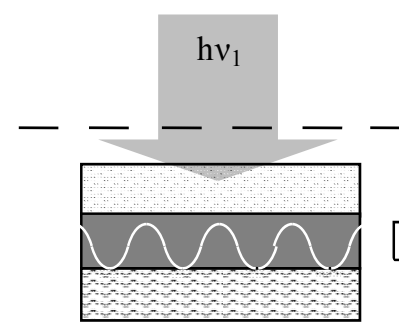

(5) 2nd Exposure

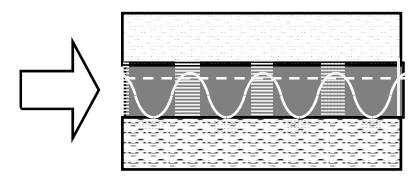

(6) Threshold Image
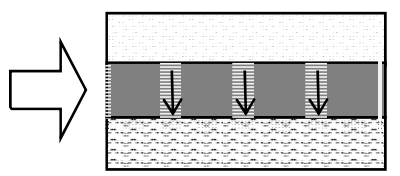

(7) Diffusion
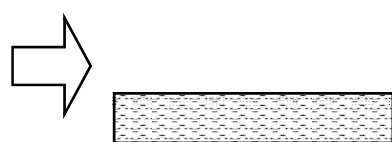

(8) Strip

Fig. 1 Mechanism of OTL 


\subsection{Materials Design}

The above approach was motivated by the work of O’Leary and Paul ${ }^{[6][7]}$, where the gas permeability of long alkyl side chain crystalline polymers, mainly poly(n-alkyl methacrylate), have been extensively investigated. It was found that the gas permeability of the melted poly(n-alkyl methacrylate) is orders of magnitude higher than that of the crystal phase. It exhibits a "jump" across the phase transition region, which shows a non-linear response of the permeability to the temperature. However, the permeability switch in this case was achieved thermally. In order for this permeability switch to be triggered by light, a light sensitive moiety must be incorporated into the crystalline long alkyl side chain region either by doping or copolymerization. Azobenzenes, a class of photochromic compounds, were chosen for the initial study of OTL system due to the large body of available literature and the ease of synthesis ${ }^{[8][9][10]}$. The designed structure is shown in Fig. 2.

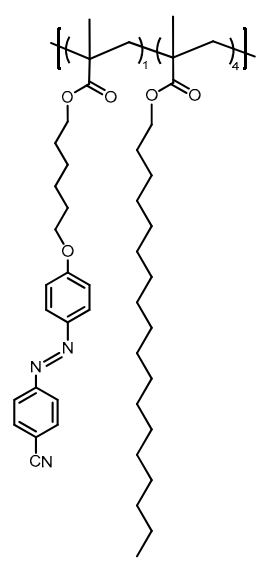

Fig. 2. The structure of the designed OTL material

The azobenzene moieties are designed to be incorporated into the long alkyl side chain region of poly(n-alkyl methacrylate). Upon UV irradiation, the nitrogen double bonds undergo cis-to-trans isomerization which disrupts the crystalline region and lowers the melting point causing a melt. Upon irradiation at a different wavelength, the cis form of azobenzene can be reversed back to its trans form and the side chain crystal reforms.

\subsection{Materials Evaluation}

A set of evaluations were carried out in order to study the performances of the new polymers. UV-Vis spectroscopy was used to confirm the photon-induced isomerizations of the azobenzene moieties. The phase transition temperatures of the material were determined by measurements on an ellipsometer equipped with a heating stage, which monitors the thickness of the polymer film while increasing the temperature. The effect of the exposure dose on the melting point was evaluated. Moreover, a trilayer diffusion experiment was designed to assess the permeability of the barrier layer. The basic feasibility of patterning through OTL was also studied. The details were provided in the following sections. 


\section{EXPERIMENTAL SECTION}

\subsection{General}

The UV-Vis absorbance spectra were collected on a Nicolet Magna-IR 500 using sub-micron films on a quartz disk. The exposures of the thin films, causing the isomerizations, were conducted with a Novacure N2001-1A lamp equipped with a light guide and exposure box. The lamp output was filtered through ORIEL band pass filters. The photon flux was measured by a Molectron POWER MAX500 photometer with a sensor area of $1 \mathrm{~cm}^{2}$. A CYMER ELS $5400 \mathrm{KrF}$ laser was used for $248 \mathrm{~nm}$ exposure. The laser intensity was measured by a Coherent Field MAX II laser power meter covered by an aluminum foil with a $1 \mathrm{~cm}^{2}$ square hole.

\subsection{Synthesis of Side Chain Crystal Polymer}

The azobenzene monomer was synthesized according to the published procedures ${ }^{[11][12][13][14]}$. The polymer was obtained by an AIBN free radical polymerization of the azobenzene monomer and octadecyl methacrylate. All the starting materials were purchased from Sigma-Aldrich and used without further purification. AIBN was recrystallized from methanol before using.

\subsection{Measurement of the melting temperatures}

The sample films were prepared by spin-coating a solution of polymer on a wafer. The films were placed on a custom built PELTIER hot plate installed on the stage of a J.A. Woollam M-2000V ellipsometer. The hot plate was controlled by a NEWPORT 3040 thermal controller. A LabVIEW program was used to control the temperature of the hot plate and synchronize the hot plate with the ellipsometer. The thickness of the polymer film was then monitored while increasing the hot plate temperature in a range which covered the melting temperature. The thickness of the polymer film was plotted as a function of temperature from which the melting temperature was determined by numerical regressions.

\subsection{Measurements of the acid diffusion coefficient}

The anisotropic diffusion of the active species through the barrier layer is the most crucial step in the OTL approach. Since the barrier layer is designed to have a low permeability in its crystalline state and a much higher permeability in its melted state, the diffusion coefficients for both states need to be quantified.

A "sandwich" or trilayer approach ${ }^{[15]}$ to determine the permeability of the polymer thin films has been designed and developed as shown in Fig. 3. Acid is considered a surrogate for the final system in which a diffusing species will cross the barrier and react with the upper most slice of the target layer. The trilayer stack consists of the polymer being studied sandwiched between an acid feeder layer and an acid detector layer. The acid passes through the barrier material and reaches the top detector layer during the baking process. The acid catalyzed reaction that occurs in the detector layer is monitored to indicate the arrival of the acid. Knowledge of this transit time and the thickness of the film allows calculation of the diffusion coefficient of the OTL materials. 


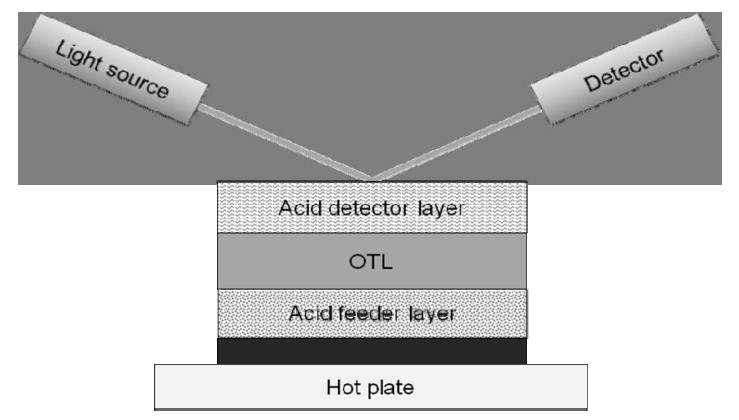

Fig. 3. The experiment setup for the acid diffusion detection in the trilayer system

Initially, poly(4-tert-butyloxycarbonyloxystyrene) (PTBOCST) was used as the acid detector. Its acid-catalyzed deprotection can be monitored by FTIR as decrease in the intensity of the carbonyl group from $1690 \mathrm{~cm}^{-1}$ to $1740 \mathrm{~cm}^{-1}$. However, the disadvantage of this measurement is that the deprotection happens above $70{ }^{\circ} \mathrm{C}$. OTL working below $70{ }^{\circ} \mathrm{C}$ cannot be tested by this method. The OTL is designed to work at room temperature; therefore, another acid detector, end-capped poly(phthalaldehyde) (PPHA), was chosen. The end-capped polymer undergoes acid-catalyzed deprotection at room temperature. PPHA has a ceiling temperature of $-40{ }^{\circ} \mathrm{C}$, and readily 'unzips' to the monomer at temperatures above $-40{ }^{\circ} \mathrm{C}$ as shown in Fig. 4 .

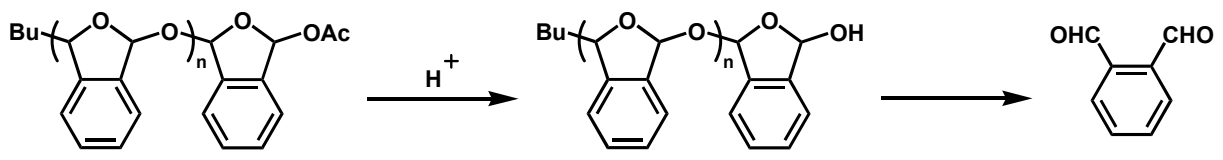

Fig. 4. The acid-catalyzed self-depolymerization of PPHA

The arrival of the acid was detected by monitoring the intensity of the reflected light from the top of the film stack. As the PPHA depolymerizes, the surface roughness of the film increases, resulting in scattering of the incident light and decreasing in the intensity of the reflected light. The diffusion coefficient of acid can be calculated from Fick's Equation:

$$
D=\frac{L^{2}}{2 t_{\text {diff }}}
$$

Where $\mathrm{L}$ is the thickness of the studied polymer layer and $t_{\text {diff }}$ is the acid transit time. The permeability of the model compound, poly(octadecyl methacrylate) (PODMA), was studied using this method. The acid feeder comprises a polymer matrix, poly(butyl methacrylate) (PBMA), and a photoacid generator, triphenyl sulfonium trifluoromethanesulfonate (TPS-OTf). A nonane solution of PODMA was spin coated on the acid feeder layer. Before being coated with the acid detector, which is PPHA, the film stack was flashed with $\mathrm{O}_{2}$ plasma in order to assure that the PPHA solution would spread on the top of PODMA without affecting the thickness. The trilayer film stack was exposed at $248 \mathrm{~nm}$ and then placed on the hot plate installed on the ellipsometer. The intensity of the reflected light on the surface of the film was monitored by ellipsometer while holding the film stack at a constant temperature. The acid transit time was found by plotting the monitored signal versus time and finding the time delay before arrival of the acid at the detector layer. The experiment was repeated at several different temperatures. 


\subsection{Patterning through a Side Chain Crystal Polymer}

The patterning approach was inspired by PRIME process ${ }^{[16]}$ in which a pattern of phenolic groups is created and silylated to give a contact mask against $\mathrm{O}_{2}$ plasma etching. Referring to this process, a trilayer imaging system was established, including a top acid feeder layer, OTL layer and a bottom imaging layer. PTBOCST was chosen as an imaging material to give phenolic groups upon acid catalyzed deprotection. In this approach, the pattern was created in the OTL layer through which the acid is able to travel at the room temperature. After the acid reaches the top of the imaging layer, it stops at the bottom layer due to the rigidity of the imaging layer. The top two layers then can be developed away. The uncovered sub layer can be further baked and silylated to incorporate silicon by exposing to HDMS vapor. The silylated regions in the sublayer can be regarded as a very thin contact mask to the anisotropic reactive ion etching. Although the acid diffusion in PTBOCST layer during PEB cannot be neglected in sub $32 \mathrm{~nm}$ imaging, the chemistry is sufficient for a proof-of-principle demonstration..

The trilayer film stack was spin coated on the wafer following the procedure previously discribed. The film was exposed to $248 \mathrm{~nm}$ laser light through a customized mask followed by a soft bake above the melting point of the barrier layer. The top two layers were then removed by cyclohexane. After PEB at $100^{\circ} \mathrm{C}$, the film was transferred to a vacuum oven to undergo vapor phase silylation (HMDS, 60 Torr, $100{ }^{\circ} \mathrm{C}, 2 \mathrm{hr}$ ). The silylated film was then etched by $\mathrm{O}_{2}$ RIE (40 $\mathrm{sccm}, 30 \mathrm{mTorr}, 120 \mathrm{~W}$ ) at room temperature. The image created was observed with an OLYMPUS BX60 microscope. The cross-section was examined by a DEKTEK 6M profilometer.

\section{RESULTS AND DISCUSSIONS}

\subsection{Isomerization of Azobenzene in the polymer thin film}

UV-Vis spectra of the azobenzene containing thin film were collected before exposure at $365 \mathrm{~nm}$ and for every 30 $\mathrm{mJ} / \mathrm{cm}^{2}$ exposure dose up to $140 \mathrm{~mJ} / \mathrm{cm}^{2}$. The spectra were shown below in Fig. 5 .
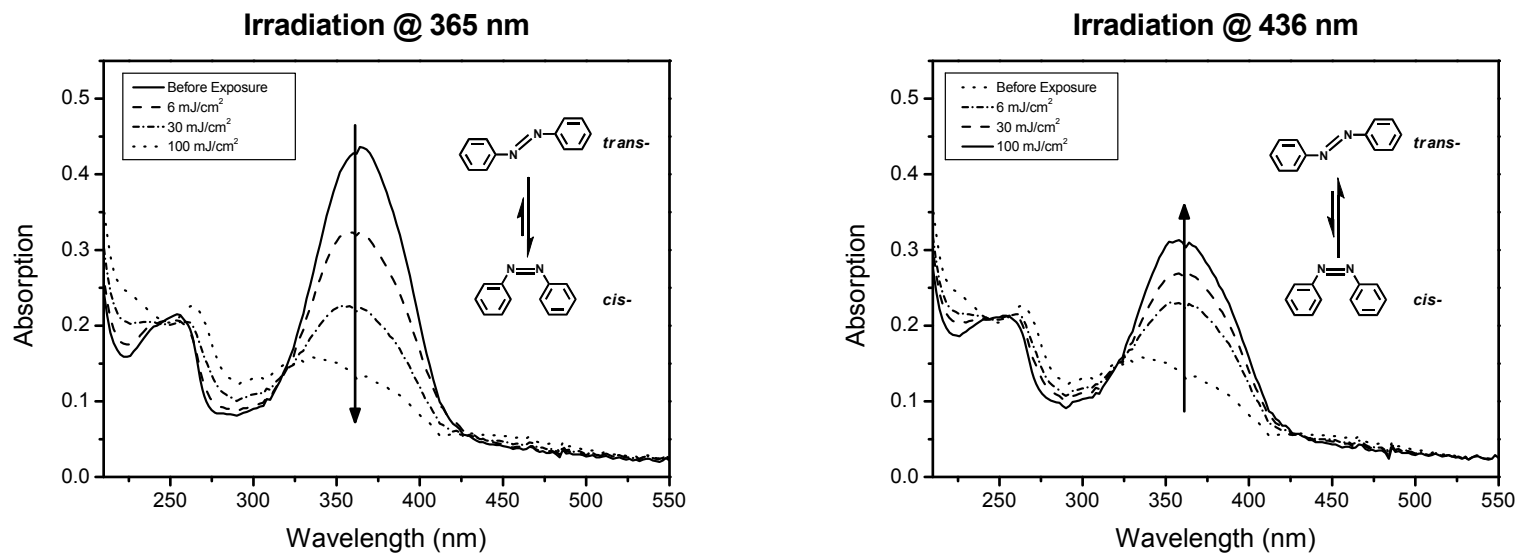

Fig. 5. UV-Vis spectra of the isomerization in the synthesized polymer 
The absorption maximum $\left(\lambda_{\max }\right)$ of the polymer is at $366 \mathrm{~nm}$. This is the absorbance of the $\pi-\pi^{*}$ transition of the trans nitrogen double bond. When the trans form is exposed to UV light at $365 \mathrm{~nm}$ which covers its $\pi-\pi^{*}$ band ${ }^{[8]}$, the azobenzene isomerizes to the cis form and the absorption peak at $366 \mathrm{~nm}$ shrinks. When the cis form of the azobenzene was exposed at $436 \mathrm{~nm}$, it isomerized back to the trans form. However, the system did not completely reverse to its original state. It is believed that it reached a photostationary state due to the overlap of the excitation bands for both cis and trans forms. This problem can be solved by blue shifting the exposure wavelength.

\subsection{Measurement of the melting temperatures of the thin films}

The melting temperature of the side chain crystal polymer was determined by a thermal mechanical analysis where the thermal expansion was investigated over a wide range of temperatures as described in the experimental section. The result for the synthesized polymer was plotted as film thickness versus temperature as shown in Fig. 6.

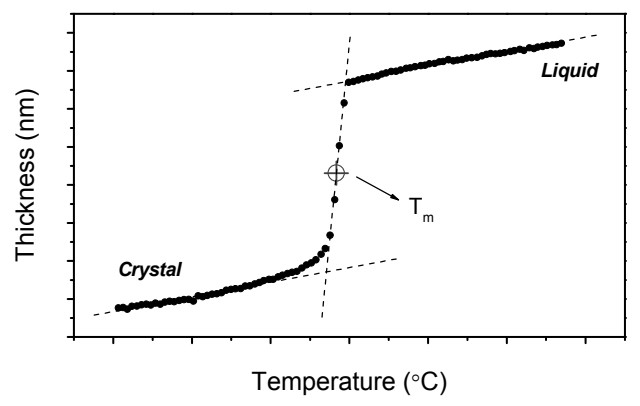

Fig. 6. The increase of thickness of the polymer thin film with increasing the temperature

The melting temperature of the thin film was extrapolated by the mathematic process described in the experimental section. Fig. 7 shows the melting temperatures of the exposed thin film measured using the same method. The figure indicates that the melting temperature of the crystal region was depressed by $2{ }^{\circ} \mathrm{C}$ and could be reversed back to near the original temperature.

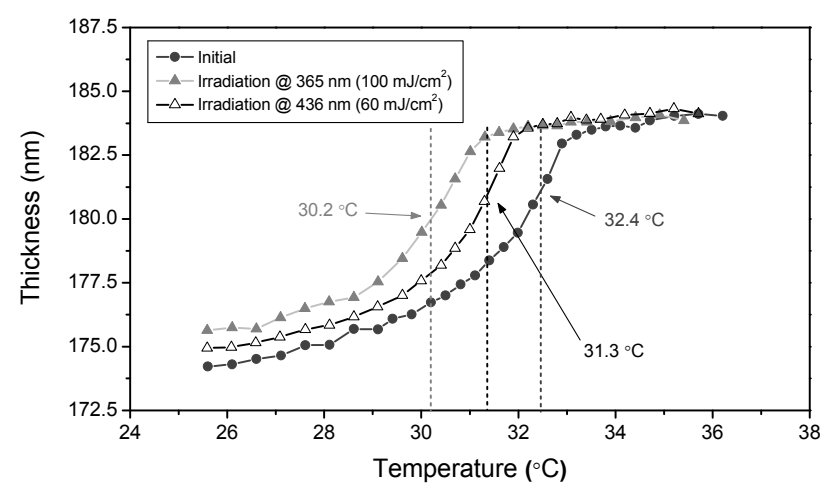

Fig. 7. Film thickness and melting point of the P122 film as function of temperature and exposure.

However, $2{ }^{\circ} \mathrm{C}$ is not large enough for an applicable photoswitch in the proposed system. The reason is that the 
melting temperature of a polymer is not an exact number but a certain range of temperatures because of the different sizes and low degree of perfection of the polymer crystals. In order to achieve a useful optical switch from crystalline to amorphous, the melting temperature needs to be shifted by at least $5{ }^{\circ} \mathrm{C}$. We believe that the azobenzene chromophore is being excluded from the crystalline region. Several modifications to the polymer have been designed to increase incorporation. These data will be reported subsequently.

\subsection{Measurement of the acid permeability}

The trilayer diffusion experiments demonstrated the delay of the acid arrival at the detector layer at different temperatures, shown in Fig. 8. The diffusion times are showed on the X-Axis where the decrease of the intensity begins.

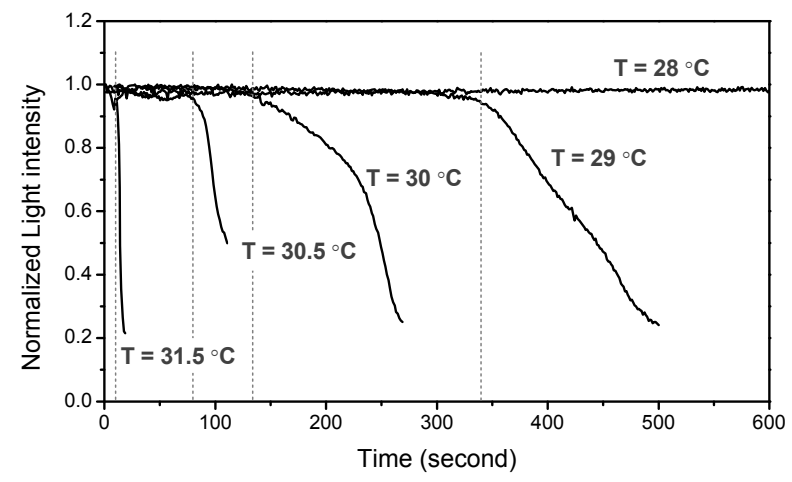

Fig. 8. Acid transport time through PODMA film as a function of temperature

According to Fickian relation (Equ. 1), the acid permeability of PODMA can be calculated from the diffusion length, which is the thickness of the barrier layer, along with the diffusion time. The acid permeability of PODMA was plotted as a function of temperature shown in Fig. 9. The results show a non linear response that is consistent with the result in O'Leary and Paul's work ${ }^{[6][7]}$.

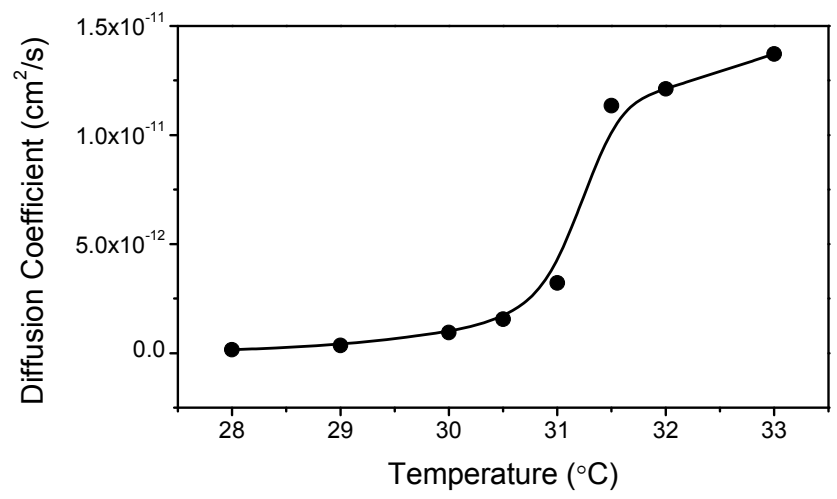

Fig. 9. The dependence of the diffusion coefficient on the temperature 


\subsection{Feasibility of patterning through side chain crystal polymer}

The patterning concept was confirmed by image transferring through PODMA. PODMA does not undergo an optically induced melting temperature shift. So the transfer of the pattern was triggered thermally by baking the film stack at a temperature that is $2{ }^{\circ} \mathrm{C}$ higher than the melting temperature. In this approach, a latent image of acid was first created in the acid feeder layer upon UV exposure. The image was subsequently transferred through the barrier layer, where the permeability was controlled, to the imaging layer. Since its ability to travel through the melted polymer has already been demonstrated, the trifluoromethane sulfonic acid was used as the diffusant to facilitate the acid catalyzed reaction in the imaging layer. After the acid traveled through the barrier layer and reached the imaging layer, the acid feeder and barrier layers were removed via wet development in cyclohexane and the imaging layer was baked to allow the acid catalyzed reaction to occur. The image obtained is shown below in Fig. 10.

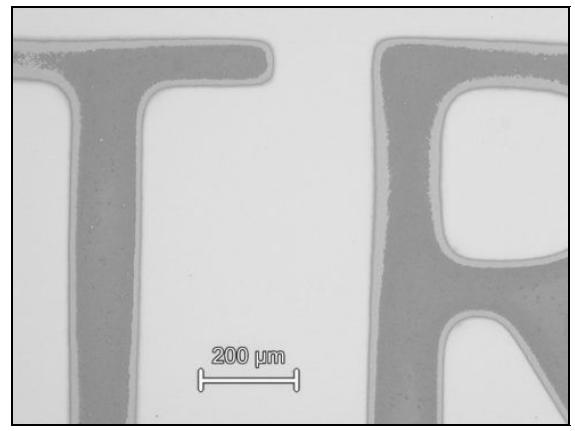

Fig. 10. Pattern created by the trilayer approach

The cross-section of image was examined by profilometer as shown in Fig. 11 and Fig. 12. This result suggests that the pattern can be transferred through a melted barrier layer by diffusion.

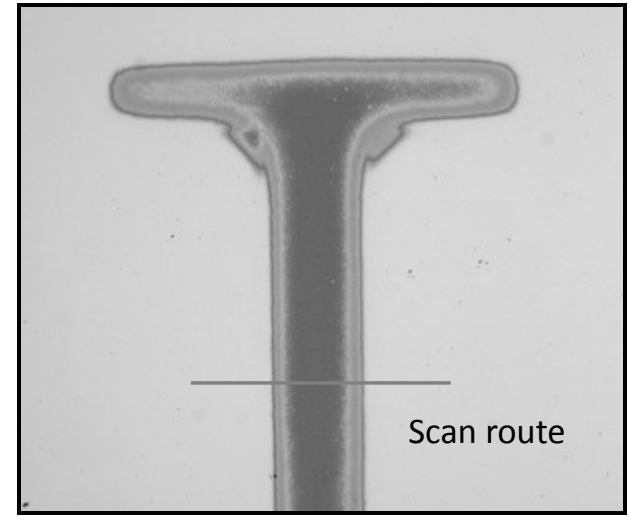

Fig. 11. The scan routine of cross-section

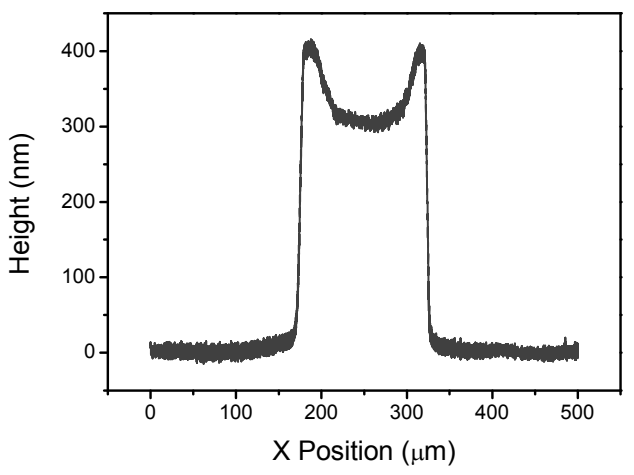

Fig. 12. The profile of the cross-section 


\section{CONCLUSION}

The principle of the OTL was established and described in this paper. A photochromic group, azobenzene, was introduced into a side chain crystal polymer to provide the necessary photoswitchable properties for application to the OTL. We have successfully demonstrated the isomerization of the azobenzene groups, the corresponding shift in the melting temperature, the modulation of the permeability and the patterning feasibility. Work is underway that is designed to provide a larger shift in the melting temperature to support the OTL system. Moreover, the azobenzenes were used for the proof-of-principle at $365 \mathrm{~nm}$, so the design of a photochromic analog of azobenzene for application at $193 \mathrm{~nm}$ is under way.

\section{ACKNOWLEDGEMENTS}

The authors would like to thank SEMATECH Engineering Research Center for funding. SEMATECH and the SEMATECH logo are registered service marks of SEMATECH, Inc. All other service marks and trademarks are the property of their respective owners.

\section{REFERENCE}

[1] T. Watanabe, and H. Kinoshita, "Current status and prospect of Extreme Ultraviolet Lithography," Journal of Photopolymer Science and Technology, 21(6), 777-784(2008).

[2] J. Benschop, V. Banine, S. Lok, and E. Loopstra, "Extreme ultraviolet lithography: Status and prospects," J. Vac. Sci. Technol. B, 26(6), 2204-2207(2008).

[3] B. S. Haran and et al., "22nm technology fully compatible fully functional $0.1 \mu \mathrm{m}^{2}$ 6T SRAM cell," International Electron Devices Meeting, 27.1.1., San Francisco, (2008).

[4] S. Lee, J. Byers, K. Jen, P. Zimmerman, B. Rice, N. J. Turro, and C. G. Willson, “An analysis of double exposure lithography options," Proc. SPIE, 6924, 69242A-1-12 (2008).

[5] J. Byers, S. Lee, K. Jen, P. Zimmerman, N. J. Turro, and C. G. Willson, "Double exposure materials: simulation study of feasibility," J. Photopolym. Sci. Technol., 20(5), 707-717 (2007).

[6] K. A. O'Leary, and D. R. Paul, "Physical properties of poly(n-alkyl acrylate) copolymers. Part 1. Crystalline / crystalline combinations," Polymer, 47, 1226-1244 (2006).

[7] K. A. O'Leary, and D. R. Paul, "Physical properties of poly(n-alkyl acrylate) copolymers. Part 2. Crystalline / non-crystalline combinations," Polymer, 47, 1245-1258 (2006).

[8] S. Xie, A. Natanshohn, and P. Rochon, "Recent developments in aromatic azo polymers research," Chem. Mater., 5(4), 403-411 (1993).

[9] A. Natansohn, "Photoinduced Motions in Azo-Containing Polymers," Chem. Rev., 102, 4139-4175 (2002).

[10] K. G. Yager, and C. J. Barrett, "Novel photo-switching using azobenzene functional materials," Journal of Photochemistry and Photobiology A: Chemistry, 182, 250-261(2006). 
[11] M. Motoi, K. Noguchi, A. Arano, S. Kanoh, and A. Ueyama, "Side chain liquid crystalline polyoxetanes with a spacer-seperated azobenzene moiety. I. preparation and characterization,” Bull. Chem. Soc. Jpn., 66(6), 1778-1789 (1993).

[12] D. E. Bergbreiter, S. D. Sung, J. Li, D. Ortiz, and P. N. Hamilton, "Designing polymers for biphasic liquid/liquid separations after homogeneous reactions," Organic Process Research \& Development, 8(3), 461-468 (2004).

[13] X. Liu, B.Yang, Y. Wang, and J. Wang, "New nanoscale pulsatile drug delivery system," Chem. Mater., 17(15), 2792-2795 (2005).

[14] C. Engels, D. V. Steenwinckel, E. Hendrickx, M. Schaerlaekens, A. Persoons, and C. Samyn, "Efficient fully functionalized photorefractive polymethacrylates with infrared sensitivity and different spacer lengths," J. Mater. Chem., 12(4), 951-957 (2002).

[15] M. Stewart, S. Postnikov, H. V. Tran, D. Medeiros, M. A. Nierode, T. Cao, J. Byers, S. Webber, and C. G. Willson, "Measurement of acid diffusivity in thin polymer films above and below $\mathrm{T}_{\mathrm{g}}$ " Proc. ACS, Polym. Mat. Sci. Eng. Div., 81(58), (1999).

[16] C. Pierrat, S. Tedesco, F. Vinet, M. Lerme, and B. Dal'Zotto, "Positive resist image by dry etching: New dry developed positive working system for electron beam and deep ultraviolet lithography," J. Vac. Sci. Technol. B, 7(6), 1782-1786 (1989). 